\section{SCIENCE CHINA \\ Physics, Mechanics \& Astronomy}

\section{-Editorial•}

Editor's Focus
May 2021 Vol. 64 No. 5: 250361

https://doi.org/10.1007/s11433-020-1650-3

\title{
Nonadiabatic geometric gates with a superconducting qubit
}

Fault-tolerant quantum computing requires high-fidelity gates so that errors can be corrected. So far, various schemes have been proposed to increase gate fidelities, including nonadiabatic holonomic gates [1-4] and topological dynamical decoupling [5]. Nonadiabatic holonomic gates have been experimentally demonstrated in nuclear magnetic resonance [1], superconducting transmon qubit systems [2], and nitrogen-vacancy centers in diamond [3,4]. As reported in ref. [2], the nonadiabatic holonomic one-qubit gates are realizable in the lowest three-level ladder-type system with the second excited state as an auxiliary level. However, owing to the relatively short coherence time of the second excited state of the transmon qubit system, the resultant gates see a reduced fidelity.

Recently, a joint collaboration between two research groups from Zhejiang University and Shandong University experimentally implementated nonadiabatic geometric gates in the cyclic evolution of a superconducting qubit system [6]. They demonstrated several specific one-qubit quantum gates: identity operator $I$, Hadamard gate $H$ and rotation gates $R_{x}(\pi), R_{x}(\pi / 2)$, $R_{y}(\pi), R_{y}(\pi / 2), R_{z}(\pi), R_{z}(\pi / 2)$, where they only used the two lowest levels of a superconducting transmon qubit, thus avoiding the influence from the short coherence time of the second excited state in ref. [2] and increasing the average gate fidelity up to $99.6 \%$. This work also indicates that the fidelity of geometric gates is higher than that of dynamic gates.

GuiLu Long Department of Physics, Tsinghua University, Beijing 100084, China

1 G. Feng, G. Xu, and G. Long, Phys. Rev. Lett. 110, 190501 (2013), arXiv: 1302.0384.

2 A. A. Abdumalikov Jr, J. M. Fink, K. Juliusson, M. Pechal, S. Berger, A. Wallraff, and S. Filipp, Nature 496, 482 (2013), arXiv: 1304.5186.

3 C. Zu, W. B. Wang, L. He, W. G. Zhang, C. Y. Dai, F. Wang, and L. M. Duan, Nature 514, 72 (2014).
4 S. Arroyo-Camejo, A. Lazariev, S. W. Hell, and G. Balasubramanian, Nat. Commun. 5, 4870 (2014).

5 J. Zhang, X.-D. Yu, G.-L. Long, and Q.-K. Xue, Sci. China-Phys. Mech. Astron. 62, 120362 (2019).

6 P. Z. Zhao, Z. J. Z. Dong, Z. X. Zhang, G. P. Guo, D. M. Tong, and Y. Yin, Sci. China-Phys. Mech. Astron. 64, 250362 (2021). 